PROCEEDINGS OF THE

AMERICAN MATHEMATICAL SOCIETY

Volume 131, Number 2, Pages 363-368

S 0002-9939(02)06550-4

Article electronically published on June 3, 2002

\title{
NILPOTENCY DEGREE OF COHOMOLOGY RINGS IN CHARACTERISTIC $p$
}

\author{
PHAM ANH MINH
}

(Communicated by Stephen D. Smith)

Dedicated to Professor Huỳnh Mùi on his sixtieth birthday

\begin{abstract}
Let $p$ be an odd prime number. The purpose of this paper is to provide a $p$-group $\mathcal{G}$ whose mod- $p$ cohomology ring has a nilpotent element $\xi \in H^{*}(\mathcal{G})$ satisfying $\xi^{p} \neq 0$.
\end{abstract}

\section{Statement of the MAin Result}

For every $p$-group $\mathcal{G}$, denote by $H^{*}(\mathcal{G})$ the mod- $p$ cohomology algebra of $\mathcal{G}$. We are now interested in the nilpotency degrees of elements of $H^{*}(\mathcal{G})$. For the case $p=2$, in 1, 4, it was shown that, given any positive integer $n$, there exists a 2 -group whose cohomology ring has elements of nilpotency degree $n+1$. It was also noted in [1] that, for $p$ odd, we did not have any example of elements of $H^{*}(\mathcal{G})$ having nilpotency degrees greater than $p$.

Recently, such an example was given in 8 for $p=3$. In this case, $\mathcal{G}$ is chosen to be an extension of $\mathbb{E} \times \mathfrak{A}$ by $\mathbb{Z} / p$, where $\mathbb{E}$ is the extraspecial 3-group of order $3^{3}$ and of exponent 3 , and $\mathfrak{A}$ the elementary abelian 3 -group of rank 4 .

The purpose of this paper is to generalize the result in 8 to the case of any odd prime $p$. Let $\mathfrak{S}_{p^{2}, p}$ be the Sylow subgroup of the symmetric group on $p^{2}$ letters (so $\mathfrak{S}_{p^{2}, p}=\mathbb{Z} / p\{\mathbb{Z} / p$, the wreath product of $\mathbb{Z} / p$ and $\mathbb{Z} / p)$ and let $Z$ be the center of $\mathfrak{S}_{p^{2}, p}$. Define $\mathfrak{R}=\mathfrak{S}_{p^{2}, p} / Z$ (so $\mathfrak{R}=\mathbb{E}$ for $p=3$ ) and let $\mathfrak{A}_{k}$ be the elementary abelian $p$-group of rank $2 k$. We shall prove

Theorem. For $k \geq 2 p-2$, there exists a p-group $\mathcal{G}$ given by a central extension

$$
0 \rightarrow \mathbb{Z} / p \rightarrow \mathcal{G} \rightarrow \mathfrak{R} \times \mathfrak{A}_{k} \rightarrow 1
$$

whose mod-p cohomology ring has a nilpotent element $\xi \in H^{2}(\mathcal{G})$ satisfying $\xi^{p} \neq 0$.

We shall use the following notation. For homogeneous elements $X, Y, \ldots$ of a graded ring $R,|X|$ denotes the degree of $X$ and $(X, Y, \ldots)$ the ideal of $R$ generated by $X, Y, \ldots p$ is assumed from now on to be an arbitrary odd prime number. If $S$ is a subset of a group $G$, then $\langle S\rangle$ denotes the subgroup of $G$ generated by $S$. With some abuse of notation, for every $\zeta \in H^{*}(G)$ and for every subgroup $K$ of $G$, we consider $\zeta$ as an element of $H^{*}(K)$ via the restriction map; also, for every extension $T$ of $G, \zeta$ is considered as an element of $H^{*}(T)$ via the inflation maps.

Received by the editors August 15, 2001 and, in revised form, September 18, 2001.

2000 Mathematics Subject Classification. Primary 20J06; Secondary 20D15, 55R40. 


\section{THE GROUPS $\mathfrak{R}$ AND $\mathfrak{U}$}

Let $\mathfrak{U}$ be the $p$-group of order $p^{p+2}$ given by

$$
\begin{aligned}
\mathfrak{U}=\left\langle a, a_{1}, \ldots, a_{p+1}\right| a^{p} & =a_{3}^{p}=\cdots=a_{p+1}^{p}=\left[a, a_{p+1}\right]=\left[a_{i}, a_{j}\right]=a_{1}^{p} a_{2}^{p(p-1) / 2} a_{p} \\
& \left.=a_{2}^{p} a_{p+1}=1,\left[a, a_{\ell}\right]=a_{\ell+1}, 1 \leq i, j \leq p+1,1 \leq \ell \leq p\right\rangle .
\end{aligned}
$$

Then $\mathfrak{U}$ is a 2-generator $p$-group of maximal class and $\mathfrak{R}=\mathfrak{U} /\left\langle a_{p}, a_{p+1}\right\rangle$ (see e.g. 2, Section 4]). Set $\mathfrak{T}=\mathfrak{U} /\left\langle a_{p+1}\right\rangle, g_{i}=a_{i}\left\langle a_{p}, a_{p+1}\right\rangle \in \mathfrak{R}, 1 \leq i \leq p-1$, and $K=\left\langle g_{1}, \ldots, g_{p-1}\right\rangle$. We then have extensions of groups

$$
\begin{array}{r}
0 \rightarrow \mathbb{Z} / p \rightarrow \mathfrak{U} \stackrel{p_{1}}{\rightarrow} \mathfrak{T} \rightarrow 1, \\
0 \rightarrow \mathbb{Z} / p \stackrel{j}{\rightarrow} \mathfrak{T} \stackrel{p_{2}}{\rightarrow} \mathfrak{R} \rightarrow 1, \\
0 \rightarrow(\mathbb{Z} / p)^{p-1} \cong K \rightarrow \mathfrak{R} \rightarrow\langle a\rangle \rightarrow 1 .
\end{array}
$$

Let $x \in H^{1}(\langle a\rangle)$ be the dual of $a$ and consider $x$ as an element of $H^{1}(\Re)$ via the inflation map. Define $x_{1} \in H^{1}(\mathfrak{R})$ satisfying $x_{1}\left(g_{1}\right)=1, x_{1}(a)=0\left(x_{1}\right.$ is welldefined, as $\mathfrak{R}$ is a 2-generator $p$-group and $\left.\mathfrak{R}=\left\langle a, g_{1}\right\rangle\right) . x, x_{1}$ is then a basis of $H^{1}(\mathfrak{R})$. Set $y=\beta x, y_{1}=\beta x_{1}$ with $\beta$ the Bockstein homomorphism. Let $w$ be the element of $H^{2}(\mathfrak{R})$ classifying the extension $(\mathfrak{T})$ and set $U=w+y_{1}$. We have

Lemma 1. (i) $\left.U\right|_{K}=0$;

(ii) $U^{p}+x y^{p-2} \cdot \beta U-y^{p-1} \cdot U=0$;

(iii) $x \cdot U=x y_{1}$;

(iv) $U^{p}=y^{p-1} y_{1}$.

Proof. Set $f=p_{1}\left(a_{1}\right)$. Since $f^{p}=j(-1)$ and $p_{2}^{-1}(K)$ is abelian, $\left.w\right|_{K}=-y_{1}$. Hence $\left.U\right|_{K}=0$. (ii) follows from [6, Remark 1] (see also [7, Theorem 1.1]) and the fact that $\left.U\right|_{K}=0$.

Note that the term $E_{2}(\mathfrak{T})$ of the Hochschild-Serre spectral sequence corresponding to the extension $(\mathfrak{T})$ is of form

$$
E_{2}(\mathfrak{T})=H^{*}(\mathfrak{R}) \otimes H^{*}(j(\mathbb{Z} / p)) .
$$

Let $w^{\prime}$ be the element of $H^{2}(\mathfrak{T})$ classifying the extension $(\mathfrak{U})$ and let $u$ be the element of $H^{1}(j(\mathbb{Z} / p))$ satisfying $d_{2}(u)=w$. Since $\left\langle a_{p}, a_{p+1}\right\rangle=p_{1}^{-1}(\operatorname{Im} j)$ is of exponent $p, w^{\prime}$ restricts trivially on $j(\mathbb{Z} / p)$. So $w^{\prime}$ represents a non-zero element of $E_{\infty}^{2,0}(\mathfrak{T}) \oplus E_{\infty}^{1,1}(\mathfrak{T})$. Note that $\left.X\right|_{\langle a, j(\mathbb{Z} / p)\rangle}$ (resp. $\left.\left.X\right|_{\left\langle a_{1}, j(\mathbb{Z} / p)\right\rangle}\right)$ is a scalar multiple of $y$ (resp. $y_{1}$ ), for any $X \in E_{\infty}^{2,0}(\mathfrak{T}) \subset \operatorname{Im~} \operatorname{Inf}_{\mathfrak{T}}^{\mathfrak{q}}$. As $\left[a, a_{p}\right]=a_{p+1}$ and $\left[a_{1}, a_{p}\right]=1$, it follows that $w^{\prime}$ represents a non-zero element $\theta$ of $E_{2}^{1,1}(\mathfrak{T})$ and $\left.w^{\prime}\right|_{\langle a, j(\mathbb{Z} / p)\rangle} \neq$ $0,\left.w^{\prime}\right|_{\langle f, j(\mathbb{Z} / p)\rangle}=0$. So $\theta$ is a non-zero scalar multiple of $x \otimes u$. This means that $x d_{2}(u)=x w=0$. Hence $x \cdot U=x\left(w+y_{1}\right)=x y_{1}$.

Finally, as

$$
\begin{aligned}
y \cdot U-x \cdot \beta U & =\beta(x \cdot U) \\
& =\beta\left(x y_{1}\right) \quad \text { by (iii) } \\
& =y y_{1},
\end{aligned}
$$


we have, by (ii),

$$
\begin{aligned}
0 & =U^{p}+x y^{p-2} \cdot \beta U-y^{p-1} \cdot U \\
& =U^{p}+y^{p-2}(x \cdot \beta U-y \cdot U) \\
& =U^{p}-y^{p-2} \cdot y y_{1} \\
& =U^{p}-y^{p-1} y_{1} .
\end{aligned}
$$

The lemma is proved.

Remarks. 1. It is known that $\operatorname{Inf}_{\mathfrak{S}_{p^{2}, p}^{\mathfrak{R}}}: H^{2}(\mathfrak{R}) \rightarrow H^{2}\left(\mathfrak{S}_{p^{2}, p}\right)$ is surjective (this comes from the fact that $\mathfrak{S}_{p^{2}, p}$ is a terminal $p$-group; see [3]). Furthermore, as $H^{*}\left(\mathfrak{S}_{p^{2}, p}\right)$ is detected by elementary abelian subgroups $([9])$ and $\left.U\right|_{\langle a\rangle}=0,\left.U\right|_{K}=$ 0 , it follows that $\operatorname{Inf}_{\mathfrak{S}_{p^{2}, p}}^{\Re}(U)=0$. Hence $U$ is nothing other than the cohomology class classifying the central extension $1 \rightarrow Z \rightarrow \mathfrak{S}_{p^{2}, p} \rightarrow \mathfrak{R} \rightarrow 1$.

2. The group $\mathfrak{U}$ can be obtained from algebraic number theory, as follows. Let $\vartheta$ be a primitive $p$ th root of unity and let $\mathbb{Z}_{p}$ be the ring of $p$-adic integers. Then

$$
\mathbb{Z}_{p}[\vartheta]=\bigoplus_{j=0}^{p-2} \vartheta^{j} \mathbb{Z}_{p}
$$

Let $\langle a\rangle$ be the cyclic group of order $p$. Multiplication by $\vartheta$ induces an action of $\langle a\rangle$ on $\mathbb{Z}_{p}[\vartheta]$, hence on $\mathfrak{Z}=\mathbb{Z}_{p}[\vartheta] /\left((\vartheta-1)^{p+1}\right)$. We can check that the map

$$
\left\{a, a_{1}, \ldots, a_{p+1}\right\} \rightarrow\langle a\rangle \ltimes \mathfrak{Z}
$$

which maps $a$ to $a, a_{i}$ to $(\vartheta-1)^{i-1}, 1 \leq i \leq p+1$, can be extended to a homomorphism of groups from $\mathfrak{U}$ to $\langle a\rangle \ltimes \mathfrak{Z}$ which is bijective. So $\mathfrak{U} \cong\langle a\rangle \ltimes \mathfrak{Z}$.

\section{Proof of the Theorem}

Pick a basis $c_{1}, \ldots, c_{2 k}$ of the elementary abelian $p$-group $\mathfrak{A}_{k}$ of rank $2 k$. Let $u_{1}, \ldots, u_{2 k}$ be the basis of $H^{1}\left(\mathfrak{A}_{k}\right)$, dual to that of $\mathfrak{A}_{k}$. Define $v_{i}=\beta u_{i}, 1 \leq i \leq 2 k$. Set $G=\mathfrak{R} \times \mathfrak{A}_{k}$. By Künneth formula,

$$
\begin{aligned}
H^{*}(G) & =H^{*}(\mathfrak{R}) \otimes H^{*}\left(\mathfrak{A}_{k}\right) \\
& =H^{*}(\mathfrak{R}) \otimes \Lambda\left[u_{1}, \ldots, u_{2 k}\right] \otimes \mathbb{F}_{p}\left[v_{1}, \ldots, v_{2 k}\right]
\end{aligned}
$$

Let

$$
\begin{aligned}
z & =y+u_{1} u_{2}+\cdots+u_{2 k-1} u_{2 k}, \\
\alpha & =u_{1} u_{2}+\cdots+u_{2 k-1} u_{2 k}, \\
\gamma & =u_{3} u_{4}+\cdots+u_{2 k-1} u_{2 k}
\end{aligned}
$$

be elements of $H^{*}(G)$. Set $\Lambda=\Lambda\left[u_{1}, \ldots, u_{2 k}\right]$ and $\Lambda^{\prime}=\Lambda\left[u_{3}, \ldots, u_{2 k}\right]$. For $i \geq 0$, let $\Lambda_{i}$ be the degree $i$-part of $\Lambda$. We have

Lemma 2. Let $0 \neq X$ be a homogeneous element of $H^{*}(G)$ and let $n, k$ be positive integers.

(i) If $n \leq \min (p-1, k)$ and $X \cdot \alpha^{n}=0$, then $|X| \geq \min (2 p-2 n, k-n+1)$.

(ii) If $X \cdot z=0$ :

(iia) $|X| \geq \min (2 p-2, k)$;

(iib) $|X|>2 p-1$, provided that $k \geq 2 p-2$ and $X \in\left(v_{1}, \ldots, v_{2 k}\right)$. 
Proof. (i) By induction on $k$. It obviously holds for $k=1$. Assume that it holds for $k-1$. Without loss of generality, we may assume that $X \in \Lambda$. If $n=k \leq p-1$, then $0=X \cdot \alpha^{n}=k ! X \cdot u_{1} \ldots u_{2 k}$ implies $|X| \geq 1$. We may then suppose that $n \leq k-1$.

Write $X=X_{1}+X_{2} \cdot u_{1} u_{2}+X_{3} \cdot u_{1}+X_{4} \cdot u_{2}$ with $X_{i}$ homogeneous in $\Lambda^{\prime}$. So $0=X \cdot \alpha^{n}=X \cdot\left(\gamma+u_{1} u_{2}\right)^{n}$ implies

$$
\begin{aligned}
X_{1} \cdot \gamma^{n} & =0 \\
\left(n X_{1}+X_{2} \cdot \gamma\right) \cdot \gamma^{n-1} & =0 \\
X_{3} \cdot \gamma^{n}=X_{4} \cdot \gamma^{n} & =0
\end{aligned}
$$

If $X_{3} \neq 0$ or $X_{4} \neq 0$, it follows from (3) and the inductive hypothesis that $\min \left(\left|X_{3}\right|,\left|X_{4}\right|\right) \geq \min (2 p-2 n, k-n)$, hence

$$
|X|=\min \left(\left|X_{3}\right|,\left|X_{4}\right|\right)+1 \geq \min (2 p-2 n+1, k-n+1) .
$$

So we may suppose that $X_{3}=X_{4}=0$. By (2) and by the inductive hypothesis, $\left|n X_{1}+X_{2} \cdot \gamma\right| \geq \min (2 p-2 n+2, k-n+1)$. Hence $|X|=\left|n X_{1}+X_{2} \cdot \gamma\right| \geq$ $\min (2 p-2 n, k-n+1)$ if $n X_{1}+X_{2} \cdot \gamma \neq 0$. If $n X_{1}+X_{2} \cdot \gamma=0$, then $n X_{1}=-X_{2} \cdot \gamma$. By (1), $X_{2} \cdot \gamma^{n+1}=0$. By the inductive hypothesis, $\left|X_{2}\right| \geq \min (2 p-2 n-2, k-n-1)$. Hence $|X|=\left|X_{2}\right|+2 \geq \min (2 p-2 n, k-n+1)$. (i) is then proved.

(ii) Write $X=\sum_{i=0}^{m} X_{i} \cdot Y_{i}$ with $Y_{i} \in \Lambda_{i}, X_{i} \in H^{*}(\Re) \otimes \mathbb{F}_{p}\left[v_{1}, \ldots, v_{2 k}\right]$ and $X_{m}$. $Y_{m} \neq 0$. Then $X_{m} \cdot Y_{m} \cdot \alpha=0$. By (i), it follows that $|X|=\left|X_{m} \cdot Y_{m}\right| \geq$ $\min (2 p-2, k)$. (iia) is proved.

Suppose that $k \geq 2 p-2,|X| \leq 2 p-1$ and $X \in\left(v_{1}, \ldots, v_{2 k}\right)$. Fix $i$ with $1 \leq i \leq 2 k$ and write $X=v_{i} X^{\prime}+X^{\prime \prime}$ with $X^{\prime \prime}$ free of $v_{i}$. Then $X^{\prime} \cdot z=0$. As $\left|X^{\prime}\right| \leq 2 p-3$, it follows from (iia) that $X^{\prime}=0$. $X$ is then free of $v_{i}$. Hence by repeating the argument, we see that $X$ is free of $v_{1}, \ldots, v_{2 k}$, a contradiction.

The lemma is proved.

Suppose from now on that $k \geq 2 p-2$. We have

Lemma 3. If $X$ is homogeneous in $H^{*}(G)$ of degree $\leq 2 p-5$ and $X \cdot \beta z \in(z)$, then $X \in(z, \beta z)$.

Proof. Write $X \cdot \beta z=A \cdot z$ with $A$ homogeneous in $H^{*}(G)$. Then $A \cdot \beta z \cdot z=0$. As $|A \cdot \beta z|=|X|+4 \leq 2 p-1$ and $A \cdot \beta z \in\left(v_{1}, \ldots, v_{2 k}\right)$, it follows from Lemma 2 (ii) that $A \cdot \beta z=0$. By [5] Lemma 2.1], $A \in\left(\beta z, u_{1} \ldots u_{2 k}\right)$. So $A \in(\beta z)$, as $|A| \leq 2 p-4<2 k$. Write $A=B \cdot \beta z$. We then have $(X-B \cdot z) \beta z=0$. Again, by [5, Lemma 2.1], $X-B \cdot z \in(\beta z)$. Therefore $X \in(z, \beta z)$. The lemma is proved.

For every $1 \leq j \leq p-1$, let $B_{j}$ be a set of elements of $\Lambda_{2 j}$ such that the disjoint union $B_{j} \sqcup\left\{\alpha^{j}\right\}$ forms a basis of $\Lambda_{2 j}$. We then have a decomposition

$$
H^{*}(\mathfrak{R}) \otimes \Lambda_{2 j}=H^{*}(\mathfrak{R}) \otimes\left(\left\langle B_{j}\right\rangle \oplus\left\langle\alpha^{j}\right\rangle\right), 1 \leq j \leq p-1 .
$$

For $1 \leq i<p-1$ and for $0 \neq b \in\left\langle B_{i}\right\rangle, \mu \in \mathbb{Z} / p$, as $\left|b-\mu \alpha^{i}\right|=2 i<2 p-2$, it follows from Lemma 2 that $\left(b-\mu \alpha^{i}\right) \cdot \alpha \neq 0$. The $B_{j}$ 's can then be chosen such that, for every $1 \leq i<p-1$,

$$
\left\{b \cdot \alpha \mid 0 \neq b \in\left\langle B_{i}\right\rangle\right\} \subset\left\langle B_{i+1}\right\rangle \text {. }
$$

Lemma 4. $y^{p-1} y_{1} \notin(z, \beta z)$. 
Proof. Note that, as $\mathfrak{R}$ is of exponent $p,\left.y^{p-1} y_{1}\right|_{\left\langle a g_{1}\right\rangle} \neq 0$. So $y^{p-1} y_{1} \neq 0$.

Suppose that $y^{p-1} y_{1}=A \cdot z+B \cdot \beta z$ with $A, B$ homogeneous in $H^{*}(G)$. Write $A=A^{\prime}+A^{\prime \prime}$ with $A^{\prime} \in H^{*}(\Re) \otimes \Lambda, A^{\prime \prime} \in\left(v_{1}, \ldots, v_{2 k}\right)$. It follows that $y^{p-1} y_{1}=A^{\prime} \cdot z$ and $A^{\prime \prime} \cdot z+B \cdot \beta z=0$. Hence, without loss of generality, we may assume that $y^{p-1} y_{1}=A \cdot z$ with $A \in H^{*}(\mathfrak{R}) \otimes \Lambda$. By the decomposition (4), we have

$$
A=A_{2 p-2}+\sum_{i=1}^{p-1}\left(A_{2 p-2 i-2} \cdot \alpha^{i}+\sum_{b \in B_{i}} A_{2 p-2 i-2, b} \cdot b\right)
$$

with $A_{j}, A_{j, b} \in H^{*}(\mathfrak{R})$. By (5), it follows that

$$
\begin{aligned}
A_{2 p-2} y & =y^{p-1} y_{1}, \\
\left(A_{2 p-2}+A_{2 p-4} y\right) \cdot \alpha & =0, \\
& \cdots \\
\left(A_{2}+A_{0} y\right) \cdot \alpha^{p-1} & =0 .
\end{aligned}
$$

Therefore, for every $i \geq 1, A_{2 i}=-A_{2 i-2} y$. So $y^{p-1} y_{1}=A_{0} y^{p}$. As $\left.y^{p-1} y_{1}\right|_{\langle a\rangle}=0$, this implies $A_{0}=0$. Hence $y^{p-1} y_{1}=0$, a contradiction. Thus $y^{p-1} y_{1} \notin(z, \beta z)$. The lemma is proved.

Let $\left\{E_{r}, d_{r}\right\}$ be the Hochschild-Serre spectral sequence corresponding to the central extension

$$
0 \rightarrow \mathbb{Z} / p \stackrel{i}{\rightarrow} \mathcal{G} \rightarrow G \rightarrow 1
$$

classified by $z \in H^{2}(G)$. Pick $s$ (resp. $\left.t\right)$ in $H^{1}(i(\mathbb{Z} / p))\left(\right.$ resp. $H^{2}(i(\mathbb{Z} / p))$ satisfying $d_{2}(s)=z\left(\operatorname{resp} . d_{3}(t)=\beta z\right)$. It is known that

$$
\begin{aligned}
& E_{2}=H^{*}(G) \otimes H^{*}(i(\mathbb{Z} / p)), \\
& E_{3}=H^{*}(G) /(z) \otimes \mathbb{F}_{p}[t] \oplus \operatorname{Ann}_{H^{*}(G)}(z) \otimes \mathbb{F}_{p}[t] s .
\end{aligned}
$$

We have

Lemma 5. There exists no element $\eta \in E_{n}^{2 p-n, n-1}, n \geq 3$, satisfying $d_{n}(\eta)=$ $y^{p-1} y_{1}$.

Proof. Suppose that $\eta$ is an element of $E_{2 r}^{2 p-2 r, 2 r-1}, r \geq 2$, represented by $X \otimes$ $t^{r-1} s \in E_{2}^{2 p-2 r, 2 r-1}$. Then $0=d_{2}\left(X \otimes t^{r-1} s\right)=X \cdot z \otimes t^{r-1}$. So $X \cdot z=0$. Since $|X|=2 p-2 r \leq 2 p-4, X=0$ by Lemma 2 . Hence $0=d_{2 r}(\eta) \neq y^{p-1} y_{1}$.

Suppose now that $\eta \in E_{2 r+1}^{2 p-2 r-1,2 r}$ is represented by $X \otimes t^{r} \in E_{3}^{2 p-2 r-1,2 r}, p-1 \geq$ $r \geq 1$. If $r=1$, then $y_{1}^{p-1} y_{2} \neq X \cdot \beta z=-d_{3}(X \otimes t)$ by Lemma 4 . If $r \geq 2$, then $-r X \cdot \beta z \otimes t^{r-1}=d_{3}\left(X \otimes t^{r}\right)=0$ in $E_{3}$. So $X \cdot \beta z \in(z)$. As $|X|=2 p-2 r-1 \leq 2 p-5$, $X \in(z, \beta z)$ by Lemma 3. This implies $X \otimes t^{r}=0$ in $E_{4}$ unless $r=p-1$ and $X \in(\beta z)$. But $r=p-1$ also implies $|X|=2 p-2 r-1=1$ and $X \in(\beta z)$, hence $X=0$. So $0=d_{2 r+1}(\eta) \neq y^{p-1} y_{1}$. The lemma is proved.

Proof of the Theorem. Set $\xi=\operatorname{Inf}_{\mathcal{G}}^{G}(U)$. By Lemma 1, $\xi^{p}=y^{p-1} y_{1}$. Since $z$ vanishes in $H^{*}(\mathcal{G}), y^{p}=-\alpha^{p}=0$ in $H^{*}(\mathcal{G})$. So $\xi^{2 p}=y^{2 p-2} y_{1}^{2}=0$. Hence $\xi$ is nilpotent. Besides, Lemmas 4 and 5 show that $y^{p-1} y_{1} \notin \operatorname{Ker} \operatorname{Inf}_{\mathcal{G}}^{G}$, which means that $\xi^{p}=y^{p-1} y_{1} \neq 0$. The Theorem is proved. 


\section{REFERENCES}

1. G.S. Avrunin, J.F. Carlson, Nilpotency degree of cohomology rings in characteristic two, Proc. Amer. Math. Soc. 118 (1993), 339-443. MR 93g:20098

2. N. Blackburn, On a special class of p-groups, Acta Math. 100 (1958), 45-92. MR 21:1349

3. L. Evens, Terminal p-groups, Illinois J. Math. 12 (1968), 682-699. MR 39:2875

4. K. Inoue, A. Kono, Nilpotency of a kernel of the Quillen map, J. Math. Kyoto Univ. 33 (1993), 1047-1055. MR 95a:20053

5. P. A. Minh, On the mod $p$ cohomology groups of extra-special p-groups, Japan. J. Math. 18 (1992), 139-154.

6. - Essential mod-p cohomology classes of p-groups: an upper bound for the nilpotency degree, Bull. London Math. Soc. 32 (2000), 285-291. MR 2001c:20106

7. Evens norm and restriction maps in mod-p cohomology of p-groups, Math. Proc. Camb. Phil. Soc. 129 (2000), 253-262. MR 2001f:20119

8. Nilpotency degree of cohomology rings in characteristic 3, Proc. Amer. Math. Soc. 130 (2002), 307-310.

9. D. Quillen, The Adams conjecture, Topology 10 (1971), 67-80. MR 43:5525

Department of Mathematics, College of Sciences, University of Hue, Dai hoc KhoA hoc, Hue, Vietnam

E-mail address: paminh@dng.vnn.vn 